\title{
ON A THEOREM OF VORONOÏ( $\left.{ }^{(}\right)$
}

\author{
BY \\ F. L. CLEAVER
}

Let $E_{n}$ denote Euclidean $n$-space; a lattice $L$ in $E_{n}$ is defined to be the totality of all points of the form $g_{1} X_{1}+g_{2} X_{2}+\cdots+g_{n} X_{n}$ where $g_{1}, g_{2}, \cdots, g_{n}$ are arbitrary integers and $X_{1}, X_{2}, \cdots, X_{n}$ is a fixed set of linearly independent points of $E_{n}$. The set $X_{1}, X_{2}, \cdots, X_{n}$ is called a basis of $L$.

Let $H$ be any sphere with the origin $O$ and at least $n$ linearly independent points of a lattice $L$ in $E_{n}$ on its boundary and no points of $L$ in its interior. The theorems proved in this paper show, for $n=3$ and 4 , what choices of points from the $n$ or more points on the boundary of $H$ form a basis of $L$, and most of the possible lattice configurations are determined. These more general results include Voroni's theorem which states that for $n=3$ and 4 and for exactly $n$ linearly independent points of the lattice $L$ on the boundary of $H$ and no points of $L$ interior to $H$ the $n$ points form a basis of $L$.

LEMMA 1. Let $L$ be a lattice in $E_{n}$ and $H$ a hypersphere with the origin $O$ and the points $P_{1}, P_{2}, \cdots, P_{m}$ of $L$ on its boundary and with no points of $L$ in its interior. Suppose also that $X_{1}, X_{2}, \cdots, X_{n}$ are any $n$ linearly independent points chosen from the set $P_{1}, P_{2}, \cdots, P_{m}$. Then the convex hull $K$ of the points $\pm X_{1}, \pm X_{2}, \cdots, \pm X_{n}$ contains no point of $L$ apart from its vertices and the origin.

Proof. Let $Q=\{X:|X| \leqq|X-Z|$ for all $Z \in L\} . Q$ is the Voronoï body [1] associated with the lattice $L$, and $Q$ is convex. If $\frac{1}{2} X_{1}$ is not in $Q$, then there exists $P \in L$ such that $\left|\frac{1}{2} X_{1}\right|>\left|\frac{1}{2} X_{1}-P\right|$, and hence $X_{1} \cdot P>P^{2}$. Let $C$ denote the center of $H$. Since $X_{1}$ is on $H$ and $P$ and $X_{1}-P$ are not interior to $H$, we have $P^{2} \geqq 2 P \cdot C, X_{1}^{2}=2 X_{1} \cdot C$ and $X_{1}^{2}-2 P \cdot X_{1}+P^{2} \geqq 2 X_{1} \cdot C-2 P \cdot C$. Substituting $X_{1}^{2}$ for $2 X_{1} \cdot C$ we obtain $P^{2}+2 P \cdot C \geqq 2 P \cdot X_{1}$, but $2 P \cdot X_{1}>2 P^{2}$ hence $2 P \cdot C>P^{2}$, a contradiction. Thus $\frac{1}{2} X_{1} \in Q$. Thus the vertices of $K$ are in $2 Q$, and, since $K$ is convex, $K$ is a subset of $2 Q$. Therefore $K$ has no points of $L$ in its interior other than $O$.

Next we show that $K$ has no points of $L$ in its boundary other than the vertices. Since the (n-1)-dimensional face determined by $X_{1}, X_{2}, \cdots, X_{n}$ is interior to $H$,

Received by the editors April 3, 1961.

(1) This paper is part of the author's Tulane Dissertation in Geometry of Numbers, and was in part supported by the National Science Foundation. I wish to express my gratitude to Professor A. C. Woods for his help and encouragement. 
except for the vertices, it cannot contain another point of $L$. By symmetry a similar argument holds for the face determined by $-X_{1},-X_{2}, \cdots,-X_{n}$. Next suppose that a lattice point $P$ is interior to an $i$-dimensional face $F$ of $K$ and we show that $O P$ is perpendicular to $F$. Let $X$ be any point near $\frac{1}{2} P$ and on $\frac{1}{2} F$ then $X=\frac{1}{2} P+Z$ for some vector $Z$. Now $X \in \frac{1}{2} K \subset Q$, hence $|X| \leqq|X-P|$, i.e., $\left|\frac{1}{2} P+Z\right| \leqq\left|-\frac{1}{2} P+Z\right|$, and we have $2 Z \cdot P \leqq 0$. But also $\frac{1}{2} P-Z \in \frac{1}{2} F$, since $\frac{1}{2} P$ is interior to $\frac{1}{2} F$. Hence $-2 Z \cdot P \leqq 0$ so $O P$ is perpendicular to $F$.

In view of the above we consider $i$-dimensional faces determined by lattice points with at least one minus sign and at least one plus sign. For example, suppose that the face $F$, determined by $-X_{1}, X_{2}, \cdots, X_{n}$, contains a point $P$ of $L$ in its interior. By the above $O P$ is perpendicular to $F$. Thus $\left(X_{2}-P\right) \cdot P=0$ and $\left(X_{1}+P\right) \cdot P=0$ which implies that $X_{2} \cdot P=P^{2}$ and $X_{1} \cdot P=-P^{2}$. Now, since $X_{2}$ is on $H$ and $P$ is not inside $H$, we have $X_{2}^{2}=2 X_{2} \cdot C$ and $P^{2} \geqq 2 P \cdot C$. Now $X_{2}-P \in L$ implies $\left(X_{2}-P-C\right)^{2} \geqq C^{2}$, hence $P^{2}-2 X_{2} \cdot P \geqq 2 P \cdot C$. But $X_{2} \cdot P=P^{2}$, thus $P^{2} \leqq 2 P \cdot C$, and we have $P^{2}=2 P \cdot C$. Now $P+X_{1} \in L$ implies $P^{2}+2 P \cdot X_{1} \geqq 2 P \cdot C$ and, since $P \cdot X_{1}=-P^{2}$, we have $-P^{2} \geqq 2 P \cdot C=P^{2}$, a contradiction. Therefore, $P$ is not interior to $F$. Clearly, a similar argument can be given for any $i$-dimensional face with two of the vertices of opposite sign, therefore the lemma is proved.

THEOREM 1. Let L denote a lattice in $E_{3}$ and $H$ a sphere with the points $O, X_{1}, X_{2}, \cdots, X_{m}$ of $L$ on its boundary and no points of $L$ in its interior. Furthermore assume that there are three linearly independent points in the set. Then either any choice of three linearly independent points is a basis for $L$, or if there exists a choice of three linearly independent points that is not a basis, then $L$ is a rectangular lattice, $H$ has eight points of $L$ in its boundary, and the center of $H$ is at the center of one of the cells of $L$.

Proof. Choose three linearly independent points $X_{1}, X_{2}, X_{3}$. By Lemma 1 , the convex hull $K$ of the points $\pm X_{1}, \pm X_{2}, \pm X_{3}$ contains no points of $L$ other than $O$ and its vertices.

LEMMA 2. Let $Y_{1}, Y_{2}, Y_{3} \in L$, where Lis a lattice in $E_{3}$, such that the convex hull of the points $\pm Y_{1}, \pm Y_{2}, \pm Y_{3}$ contains no point of $L$ apart from its vertices and $O$; then either $Y_{1}, Y_{2}, Y_{3}$ is a basis of $L$ or $Y_{1}, Y_{2},\left(Y_{3}-Y_{1}-Y_{2}\right) / 2$ is a basis of $L$.

This lemma is a well-known result proved by Minkowski [2].

Since $K$ satisfies the hypotheses of Lemma 2, either $\left\{X_{1}, X_{2}, X_{3}\right\}$ or $\left\{X_{1}, X_{2},\left(X_{3}-X_{1}-X_{2}\right) / 2\right\}$ is a basis of $L$. If $X_{1}, X_{2}, X_{3}$ is a basis the theorem follows, so assume that $X_{1}, X_{2},\left(X_{3}-X_{1}-X_{2}\right) / 2$ is a basis. Let $C$ denote the center of $H$, then $\left|X_{i}-C\right|=|C|$ hence $X_{i}^{2}=2 X_{i} \cdot C$ for $i=1,2, \cdots, m$. Let $Y_{1}=\left(X_{1}+X_{2}+X_{3}\right) / 2, \quad Y_{2}=\left(-X_{1}+X_{2}+X_{3}\right) / 2, \quad Y_{3}=\left(X_{1}-X_{2}+X_{3}\right) / 2$, 
and $Y_{4}=\left(X_{1}+X_{2}-X_{3}\right) / 2$; then $Y_{i} \in L$ for $i=1,2,3,4$ and $\left(Y_{i}-C\right)^{2} \geqq C^{2}$, hence $Y_{i}^{2} \geqq 2 Y_{i} \cdot C$. Now,

so that

$$
\sum_{i=1}^{4} Y_{i}^{2}=\sum_{i=1}^{3} X_{i}^{2}, \text { and } \sum_{i=1}^{4} Y_{i}=\sum_{i=1}^{3} X_{i},
$$

$$
\sum_{i=1}^{3} X_{i}^{2}=\sum_{i=1}^{4} Y_{i}^{2} \geqq 2 \sum_{i=1}^{4} Y_{i} \cdot C=2 \sum_{i=1}^{3} X_{i} \cdot C=\sum_{i=1}^{3} X_{i}^{2} .
$$

Thus equality holds throughout, and $Y_{i}$ is on $H$ for $i=1,2,3,4$. Therefore, $L$ has eight points on the boundary of $H$ and $L$ must be a rectangular lattice, since $O, X_{1}, Y_{3}$, and $Y_{4}$ are the vertices of a parallelogram inscribed in a circle. The same is true of the points $O, Y_{2}, X_{3}, Y_{3}$ and $O, Y_{2}, X_{2}, Y_{4}$. Thus $Y_{2}, Y_{3}$, and $Y_{4}$ are mutually perpendicular and are a basis for $L$, and $C$ is the center of the cell determined by $O, Y_{2}, Y_{3}, Y_{4}$.

We have the following corollary to this theorem.

CoRollary 1. If $H$ has five or more and less than eight points of $L$ on its boundary then there exist at least two lattice vectors that are perpendicular, moreover there are three possible lattice configurations which we call the 5-configuration, the 6-configuration, and the rhombus.

Proof. Let $\pi$ be a plane tangent to $H$ at $O$. Then, keeping $O$ in $\pi$, rotate $\pi$ into $H$ until $\pi$ contains two linearly independent points, say $X_{1}$ and $X_{2}$, of $L \cap H$. If there is a fourth point of $L \cap H$ in $\pi$ then the four points are the vertices of a parallelogram inscribed in a circle, and we may choose $X_{1}$ and $X_{2}$ so that $X_{1}+X_{2}$ is the fourth point and $O X_{1}$ is perpendicular to $O X_{2}$. Let $X_{3}$ be any point of $L \cap H$ not in $\pi$; then, using Theorem 1 , we know that $X_{1}, X_{2}, X_{3}$ are a basis of $L$. Let $\pi^{\prime}$ denote the plane through $X_{3}$ parallel to $\pi$, and suppose there is a point $Y$ of $L \cap H$ that is not in $\pi$ and not in $\pi^{\prime}$. Then $Y$ must be on the same side of $\pi$ as $\pi^{\prime}$ by the choice of $\pi$. By Theorem $1, X_{1}, X_{2}, Y$ are a basis for $L$, and the determinants of $X_{1}, X_{2}, X_{3}$ and $X_{1}, X_{2}, Y$ and of $L$ are equal. Hence, the perpendicular distance of $X_{3}$ from $\pi$ is equal to the perpendicular distance of $Y$ from $\pi$, therefore $Y$ is in $\pi^{\prime}$ contrary to our assumption. Therefore all points of $L \cap H$ must lie in $\pi$ or $\pi^{\prime}$.

We now consider two cases. Suppose first that $\pi$ contains the four points $O, X_{1}, X_{2}, X_{1}+X_{2}$, then if $\pi^{\prime}$ has only one point of $L \cap H$ we call this configuration the 5-configuration for future reference. If $\pi^{\prime}$ has two points, say $X_{3}$ and $X_{4}$, then the line joining $X_{3}$ and $X_{4}$ is parallel to one of the sides of the rectangle in $\pi$, because the lattice configuration in $\pi^{\prime}$ is the same as the 2-dimensional sublattice in $\pi$. Suppose it is parallel to $O X_{1}$, then the plane through $O, X_{1}, X_{3}$ and $X_{4}$ has four points of $L \cap H$, therefore $O X_{3}$ is perpendicular to $O X_{1}$. We may think of this configuration as having three sides rectangles and ends triangles. We call 
this configuration the 6-configuration for future reference. It is impossible to have three points of $L \cap H$ in $\pi^{\prime}$, since the lattice configuration in $\pi^{\prime}$ is a copy of the configuration in $\pi$ and we would have eight points in $L \cap H$.

Next we consider the case where $\pi$ has only three points of $L \cap H$, say $O, X_{1}, X_{2}$. Now $\pi^{\prime}$ must have at least two points of $L \cap H$; suppose $X_{3}$ and $X_{4}$ are the only two points. Then, since the lattice configuration in $\pi^{\prime}$ is a copy of the configuration in $\pi, X_{3} X_{4}$ is parallel to (1) $O X_{1}$ or (2) $O X_{2}$ or (3) $X_{1} X_{2}$. If (1) holds let $\delta$ be the plane through $O, X_{1}, X_{3}$ and $X_{4}$, then $X_{3} \cdot X_{1}=0$ or $X_{4} \cdot X_{1}=0$. If (2) holds let $\delta$ be the plane through $O, X_{2}, X_{3}$ and $X_{4}$, again $X_{3} \cdot X_{2}=0$ or $X_{4} \cdot X_{2}=0$. If (3) holds let $\delta$ be the plane through $X_{1}, X_{2}, X_{3}$ and $X_{4}$, again we have a rectangle in $\delta$. In each of these three cases we have the 5-configuration.

If $\pi^{\prime}$ contains three points, say $X_{3}, X_{4}$ and $X_{5}$, then we have a triangle inscribed in $\pi^{\prime} \cap H$. If the sides of this triangle are parallel to the sides of the triangle in $\pi$, then we have the 6-configuration. Since the lattice configuration in $\pi^{\prime}$ is the same as that in $\pi$, it is possible for the three points in $\pi^{\prime}$ to form a triangle which has its sides parallel to the triangle obtained in $\pi$ be reflecting $O, X_{1}$, and $X_{2}$ through the center of $\pi \cap H$. Label the points of $\pi^{\prime}$ so that $X_{3}$ is opposite the reflected $O, X_{4}$ opposite the reflected $X_{2}$ and $X_{5}$ opposite the reflected $X_{1}$. Then $X_{4} X_{5}$ is parallel to $X_{1} X_{2}$. Let $\delta$ be the plane through $X_{1}$, $X_{2}, X_{4}$, and $X_{5}$, then we have a rectangle inscribed in $H \cap \delta$, and we have perpendicular lattice vectors. $O$ and $X_{3}$ are on opposite sides of $\delta$ and we have a rhombus shaped configuration, which we will refer to as the rhombus. It is impossible for $\pi^{\prime}$ to have more than three points of $L \cap H$, for if it did we would have a parallelogram inscribed in $\pi^{\prime} \cap H$ and this would require four points of $L \cap H$ in $\pi$. This completes the proof of the corollary.

Next we turn our attention to the case where $n=4$, and we prove the following theorem:

THEOREM 2. Let L be a lattice in $E_{4}$ and $H$ a hypersphere with the points $O, X_{1}, X_{2}, \cdots, X_{m}$ of $L$ on its boundary and no points of $L$ in its interior. Assume that there are four linearly independent points in the set $X_{1}, X_{2}, \cdots, X_{m}$. Then:

(1) If $m=4$, then $X_{1}, X_{2}, X_{3}, X_{4}$ is a basis of $L$,

(2) If $m>4$, there exist parallel hyperplanes $K$ and $K^{\prime}$ such that all the points of $L \cap H$ lie in these two hyperplanes,

(3) If $m=8$, either we have a 3-dimensional rectangular sublattice or we can choose parallel hyperplanes $K$ and $K^{\prime}$ so that $K$ has the 3-dimensional 6-configuration with the remaining three points in $K^{\prime}$; moreover, the three points in $K^{\prime}$ determine a triangle congruent to the triangle in $K$ with respect to Lor else they are congruent to the three points of the triangle in $K$ reflected through the center of the 2-dimensional cross section of $H$ containing the triangle,

(4) If $m \geqq 9$, then there exists a hyperplane with eight points of $L \cap H$, and consequently a 3-dimensional rectangular sublattice of $L$. 
Proof. Suppose first that $m=4$, then $X_{1}, X_{2}, X_{3}, X_{4}$ are linearly independent points of $L$. Let $K$ denote the convex hull of the points $\pm X_{1}, \pm X_{2}$, $\pm X_{3}, \pm X_{4}$. Applying Lemma 1 we know that $K$ contains no points of $L$ other than $O$ and its vertices.

LeMma 3. Let $X_{1}, X_{2}, X_{3}, X_{4}$ be four linearly independent points of a lattice $L$ in $E_{4}$ such that the convex hull of the points $\pm X_{1}, \pm X_{2}, \pm X_{3}, \pm X_{4}$ contains no points of $L$ apart from its vertices and $O$. For $i=1,2,3,4$ put $e_{1}= \pm 1$. Then by renumbering $X_{1}, X_{2}, X_{3}, X_{4}$ if necessary, we may take $X_{1}, X_{2}, X_{3}$ together with one of the points

$$
\begin{array}{cc}
X_{4}, \quad\left(e_{2} X_{2}+e_{3} X_{3}+e_{4} X_{4}\right) / 2, & \left(e_{1} X_{1}+e_{2} X_{2}+e_{3} X_{3}+e_{4} X_{4}\right) / 2, \\
\left(e_{1} X_{1}+e_{2} X_{2}+e_{3} X_{3}+e_{4} X_{4}\right) / 3, & \left(2 e_{1} X_{1}+e_{2} X_{2}+e_{3} X_{3}+e_{4} X_{4}\right) / 4 \\
\left(2 e_{1} X_{1}+2 e_{2} X_{2}+e_{3} X_{3}+e_{4} X_{4}\right) / 5
\end{array}
$$

as a basis of $L$.

This lemma restates a theorem of Brunngraber [3]. For a proof the reader is referred to Wolff [4].

$K$, defined above, satisfies the hypotheses of this lemma. Therefore, we may apply Lemma 3 and renumbering $X_{1}, X_{2}, X_{3}, X_{4}$, if necessary, we have that $X_{1}, X_{2}, X_{3}$ along with one of the points listed in the conclusion of the lemma must form a basis of $L$. If this fourth point is $X_{4}$, the theorem is proved. So we assume one of the points other than $X_{4}$ yields a basis. We will show that we obtain a contradiction if we use any point other than $X_{4}$, which will establish (1) of the conclusion of the theorem.

Without loss of generality we may assume $e_{1}=1$ everywhere, since if $X$ is an element of a basis of a lattice $L$ then substitution of $-X$ for $X$ yields another basis of $L$.

Suppose that $X_{1}, X_{2}, X_{3}$, and $Y=\left(X_{2}+e_{3} X_{3}+e_{4} X_{4}\right) / 2$ is a basis of $L$. Let $Y_{1}=\left(X_{2}+X_{3}+X_{4}\right) / 2, Y_{2}=\left(-X_{2}+X_{3}+X_{4}\right) / 2, Y_{3}=\left(X_{2}-X_{3}+X_{4}\right) / 2$ and $Y_{4}=\left(X_{2}+X_{3}-X_{4}\right) / 2$, then $Y_{i} \in L$ but $Y_{i}$ is not on $H$ for $i=1,2,3,4$. Let $H$ have center $C$ then $\left(Y_{i}-C\right)^{2}>C^{2}$ thus $Y_{i}^{2}>2 Y_{i} \cdot C$. Each $X_{i}$ is on $H$ thus $X_{i}^{2}=2 X_{i} \cdot C$ for $i=1,2,3,4$, moreover $\sum_{i=1}^{4} Y_{i}=\sum_{i=2}^{4} X_{i}$. Thus we have

$$
\sum_{i=2}^{4} X_{i}^{2}=\sum_{i=1}^{4} Y_{i}^{2}>2 \sum_{i=1}^{4} Y_{i} \cdot C=2 \sum_{i=2}^{4} X_{i} \cdot C=\sum_{i=2}^{4} X_{i}^{2},
$$

a contradiction.

Suppose now that $Y=\left(2 X_{1}+e_{2} X_{2}+e_{3} X_{3}+e_{4} X_{4}\right) / 4$ is the point which yields a basis. Now $2 Y-X_{1}=\left(e_{2} X_{2}+e_{3} X_{3}+e_{4} X_{4}\right) / 2 \in L$, and adding $X_{i}$ for $i=2,3$, or 4 if necessary we have the point $Y_{1}=\left(X_{2}+X_{3}+X_{4}\right) / 2 \in L$. The argument given above holds and we have a contradiction. 
Next suppose that $Y=\left(X_{1}+e_{2} X_{2}+e_{3} X_{3}+e_{4} X_{4}\right) / 2$ is the point which yields a basis. Let $Y_{i}=\left( \pm X_{1} \pm X_{2} \pm X_{3} \pm X_{4}\right) / 2$ where each $Y_{i}$ has one minus and three plus signs, then $Y_{i} \in L$ and $Y_{i}$ is not on $H$ for $i=1,2,3,4$. As above we have

$$
\sum_{i=1}^{4} X_{i}^{2}=\sum_{i=1}^{4} Y_{i}^{2}>2 \sum_{i=1}^{4} Y_{i} \cdot C=2 \sum_{i=1}^{4} X_{i} \cdot C=\sum_{i=1}^{4} X_{i}^{2},
$$

a contradiction.

Suppose now that $Y=\left(X_{1}+e_{2} X_{2}+e_{3} X_{3}+e_{4} X_{4}\right) / 3$ yields a basis of $L$. Then $Y_{1}=\left(X_{1}+p_{2} X_{2}+p_{3} X_{3}+p_{4} X_{4}\right) / 3$ belongs to $L$ where $p_{i}=1$ or 2 for $i=2,3,4$, and $Y_{1}$ is obtained by adding $X_{i}$ for $i=2,3,4$ if necessary. We have $Y_{i} \cdot Y_{i}>2 Y_{i} \cdot C$, where

$$
\begin{aligned}
& Y_{2}=\frac{1}{3}\left(-2 X_{1}+p_{2} X_{2}+p_{3} X_{3}+p_{4} X_{4}\right), \\
& Y_{3}=\frac{1}{3}\left(X_{1}+\left(p_{2}-3\right) X_{2}+p_{3} X_{3}+p_{4} X_{4}\right), \\
& Y_{4}=\frac{1}{3}\left(X_{1}+p_{2} X_{2}+\left(p_{3}-3\right) X_{3}+p_{4} X_{4}\right), \\
& Y_{5}=\frac{1}{3}\left(X_{1}+p_{2} X_{2}+p_{3} X_{3}+\left(p_{4}-3\right) X_{4}\right) .
\end{aligned}
$$

The objective is to find coefficients $a_{i} \geqq 0$ so that $\sum_{i=1}^{5} a_{i} Y_{i}^{2}$ has no crossproducts, so that we require

$$
\begin{aligned}
p_{i}\left(a_{1}+a_{3}+a_{4}+a_{5}-2 a_{2}\right) & =3 a_{i+1}, \text { for } i=2,3,4, \\
p_{2} p_{3}\left(a_{1}+\cdots+a_{5}\right) & =3\left(a_{4} p_{2}+a_{3} p_{3}\right), \\
p_{2} p_{4}\left(a_{1}+\cdots+a_{5}\right) & =3\left(a_{5} p_{2}+a_{3} p_{4}\right), \\
p_{3} p_{4}\left(a_{1}+\cdots+a_{5}\right) & =3\left(a_{5} p_{3}+a_{4} p_{4}\right) .
\end{aligned}
$$

There are essentially four cases to consider:

(1) All $p_{i}=1$. Then we take $a_{1}=2$, and all other $a_{i}=1$.

(2) $p_{2}=2, p_{3}=p_{4}=1$. Then $a_{3}=2$, all other $a_{i}=1$.

(3) $p_{2}=1, p_{3}=p_{4}=2$. Then $a_{1}=0, a_{2}=a_{3}=1, a_{4}=a_{5}=2$.

In each of these cases we have that $\sum_{i=1}^{5} a_{i} Y_{i}^{2}=2 \sum_{i=1}^{5} a_{i} Y_{i} \cdot C$, which contradicts the given inequalities. The fourth case, when all $p_{i}=2$, we use the lattice point $\left(2 X_{1}+X_{2}+X_{3}+X_{4}\right) / 3$ instead of $Y_{1}$ above; and, for the corresponding inequalities, use the coefficients $1,2,1,1,1$ to get the contradiction.

Suppose now that $Y=\left(2 X_{1}+2 e_{2} X_{2}+e_{3} X_{3}+e_{4} X_{4}\right) / 5$ yields a basis of $L$. If $e_{2}=1$, we have, by adding $X_{3}$ or $X_{4}$ if necessary, $Y^{\prime}=\left(2 X_{1}+2 X_{2}+p_{3} X_{3}+p_{4} X_{4}\right) / 5$ $\in L$ where $p_{i}=1$ or 4 for $i=3,4$. If $e_{2}=-1$ we add $X_{2}$ and, if necessary $X_{3}$ or $X_{4}$, then $Y^{\prime \prime}=\left(2 X_{1}+3 X_{2}+q_{3} X_{3}+q_{4} X_{4}\right) / 5 \in: L$ where $q_{i}=1$ or 4 for $i=3,4$. We now consider the eight possibilities for the $p_{i}$ 's and $q_{i}$ 's. 
Because of the lengthy computations involved, for the rest of this proof we adopt a labeling device. If we are considering a possible lattice point, say $Z$, then we have the following five inequalities:

$$
(Z-C)^{2}>C^{2}, \quad\left(Z-X_{i}-C\right)^{2}>C^{2} \text { for } i=1,2,3,4 \text {. }
$$

We label these expanded inequalities respectively (a), (b), (c), (d), and (e), where $X_{i}^{2}$ is substituted for $2 X_{i} \cdot C$. For example, if $p_{3}=p_{4}=1$, then $Y^{\prime}=\left(2 X_{1}+2 X_{2}+X_{3}+X_{4}\right) / 5$ and the expanded inequalities are

$$
4 X_{1} X_{2}+2 X_{1} X_{3}+2 X_{1} X_{4}+2 X_{2} X_{3}+2 X_{2} X_{4}+X_{3} X_{4}
$$

$$
-3 X_{1}^{2}-3 X_{2}^{2}-2 X_{3}^{2}-2 X_{4}^{2}>0 \text {, }
$$

(c)

$$
\begin{aligned}
-6 X_{1} X_{2}-3 X_{1} X_{3}-3 X_{1} X_{4}+2 X_{2} X_{3} & +2 X_{2} X_{4}+X_{3} X_{4} \\
& +12 X_{1}^{2}-3 X_{2}^{2}-2 X_{3}^{2}-2 X_{4}^{2}>0,
\end{aligned}
$$

$-6 X_{1} X_{2}+2 X_{1} X_{3}+2 X_{1} X_{4}-3 X_{2} X_{3}-3 X_{2} X_{4}+X_{3} X_{4}$

$$
-3 X_{1}^{2}+12 X_{2}^{2}-2 X_{3}^{2}-2 X_{4}^{2}>0 \text {, }
$$

$$
\begin{aligned}
4 X_{1} X_{2}-8 X_{1} X_{3}+2 X_{1} X_{4}-8 X_{2} X_{3} & +2 X_{2} X_{4}-4 X_{3} X_{4} \\
& -3 X_{1}^{2}-3 X_{2}^{2}+18 X_{3}^{2}-2 X_{4}^{2}>0 \\
4 X_{1} X_{2}+2 X_{1} X_{2}-8 X_{1} X_{4}+2 X_{2} X_{3}- & 8 X_{2} X_{4}-4 X_{3} X_{4} \\
& -3 X_{1}^{2}-3 X_{2}^{2}-2 X_{3}^{2}+18 X_{4}^{2}>0
\end{aligned}
$$

Now (b) + (c) yields

$$
\begin{aligned}
-12 X_{1} X_{2}-X_{1} X_{3}-X_{1} X_{4}-X_{2} X_{3}- & X_{2} X_{4}+2 X_{3} X_{4} \\
& +9 X_{1}^{2}+9 X_{2}^{2}-4 X_{3}^{2}-4 X_{4}^{2}>0,
\end{aligned}
$$

and $(\mathrm{d})+(\mathrm{e})$ yields

$$
\begin{aligned}
4 X_{1} X_{2}-3 X_{1} X_{3}-3 X_{1} X_{4}-3 X_{2} X_{3} & -3 X_{2} X_{4}-4 X_{3} X_{4} \\
& -3 X_{1}^{2}-3 X_{2}^{2}+8 X_{3}^{2}+8 X_{4}^{2}>0 .
\end{aligned}
$$

But $(\mathrm{f})+(\mathrm{g})$ is the negative of (a) which is impossible.

If $p_{3}=1$ and $p_{4}=4$, then add (a) and (d) to get (f) and (b) and (c) to get (g). But $(\mathrm{f})+(\mathrm{g})$ is the negative of (e) which is impossible. If $p_{3}=4$ and $p_{4}=1, \mathrm{a}$ similar argument yields a contradiction.

If $p_{3}=p_{4}=4$, then $Z=\left(3 X_{1}+3 X_{2}+X_{3}+X_{4}\right) / 5 \in L$ and we have inequalities (a) through (e) for $Z$. Add (b) and (c) to get (f) and (d) + (e) to get (g). Then (f) $+(\mathrm{g})$ is the negative of $(\mathrm{a})+(\mathrm{b})+(\mathrm{c})$. This completes the possible values for the $p_{i}$ 's. 
If $q_{3}=q_{4}=1, Z=2 Y^{\prime \prime}-X_{2} \in L$ and for this point $Z$ add (a) and (c) to get (f) and (d) $+(\mathrm{e})$ to get $(\mathrm{g})$. Then (f) $+(\mathrm{g})$ is the negative of (b). If $q_{3}=q_{4}=4$, then $Z=3 Y^{\prime \prime}-X_{1}-X_{2}-2 X_{3}-2 X_{4} \in L$. Add (a) and (b) to get (f) and (d) $+($ e) to get $(g)$. Then (f) $+(\mathrm{g})$ is the negative of (c).

If $q_{3}=4$ and $q_{4}=1$, we must consider four points and four sets of inequalities. Let $Y^{\prime \prime}=Z_{1}, \quad Z_{2}=2 Y^{\prime \prime}-X_{2}-X_{3}, \quad Z_{3}=3 Y^{\prime \prime}-X_{1}-X_{2}-2 X_{3}$, and $Z_{4}=4 Y^{\prime \prime}-X_{1}-2 X_{2}-3 X_{3}$, and we subscript the labels of the inequalities. Add $\left(c_{1}\right)$ and $\left(d_{1}\right)$ to get $\left(h_{1}\right),\left(b_{2}\right)+\left(d_{2}\right)$ to get $\left(h_{2}\right),\left(c_{3}\right)+\left(e_{3}\right)$ to get $\left(h_{3}\right)$, and $\left(b_{4}\right)+\left(e_{4}\right)$ to get $\left(h_{4}\right)$. Then adding $\left(h_{1}\right),\left(h_{2}\right),\left(h_{3}\right)$ and $\left(h_{4}\right)$ and dividing by 5 we have (f). Next add $\left(d_{3}\right)$ and $\left(c_{4}\right)$ to get $\left(k_{1}\right)$ and $\left(b_{1}\right)+\left(e_{2}\right)$ to get $\left(k_{2}\right)$. Then $\left(\mathrm{k}_{1}\right)+\left(\mathrm{k}_{2}\right)$ is the negative of $(\mathrm{f})$, a contradiction.

If $q_{3}=1$ and $q_{4}=4$, we can give an argument similar to the above. Thus we have eliminated all the points from the conclusion of Lemma 3, except $X_{4}$, and we have proved conclusion (1) of the theorem.

Suppose now that $m>4$, and let $Q$ denote the convex hull of the points $O, X_{1}, X_{2}, \cdots, X_{m}$. Let $K$ denote a hyperplane through $O$ containing a 3 -dimensional face of $Q$. Then $K$ must contain three linearly independent points, say $X_{1}, X_{2}, X_{3}$. Now $K$ does not contain all the points of $L \cap H$, therefore the remaining points must be to one side of $K$. Let the $w$-axis be perpendicular to $K$ through $O$ and choose the positive direction of the $w$-axis to be the same as the direction in which are the remaining points of $L \cap H$ with respect to $K$. Let $X_{4}$ be any point of $L \cap H$ that is not in $K$, then $X_{1}, X_{2}, X_{3}$ and $X_{4}$ are linearly independent. Let $R$ denote the convex hull over the points $\pm X_{1}, \pm X_{2}$, $\pm X_{3}$, and $\pm X_{4}$. Then, applying Lemma $1, R$ contains no point of $L$ apart from $O$ and the vertices. Thus the hypotheses of Lemma 3 are satisfied and we have the various possibilities for bases of $L$. Suppose first that when we apply Lemma 3 we do not need to renumber $X_{1}, X_{2}, X_{3}, X_{4}$, then for each of the possible bases for $L$ we use the same inequalities that were used above for the case $m=4$ only now we have greater than or equal to zero rather than strictly greater than zero. For each case we used a hypershpere with center $C+X_{4}$ where $C$ is the center of $H$. Adding the inequalities as before we obtain $0 \geqq 0$, therefore, equality must hold in each. This means that in each case we get a lattice point on $H$ of the form $\left(a_{1} X_{1}+a_{2} X_{2}+a_{3} X_{3}-a_{3} X_{4}\right) / p$ where the $a_{i}$ 's are positive and $p=2,3,4$ or 5 . But this contradicts our choice of $K$ since a lattice point of this form is in the negative direction of the $w$-axis. Thus again each of the possible bases in Lemma 3 are eliminated except $X_{1}, X_{2}, X_{3}, X_{4}$ which must be a basis of $L$. If $X_{1}, X_{2}, X_{3}, X_{4}$ need to be renumbered, suppose that $X_{2}, X_{3}, X_{4}$ are in $K$ and $X_{1}$ is not in $K$. Then repeat the argument just given with the exception that we must note that in each case we used a hypersphere with center $P+X_{1}$ and we obtain a similar contradiction. Thus we have shown that $X_{1}, X_{2}, X_{3}, X_{4}$ is a basis for $L$. 
Let $K^{\prime}$ denote the hyperplane parallel to $K$ through $X_{4}$, then since our choice of $X_{4}$ was arbitrary we know that all the points of $L \cap H$ must lie in the two hyperplanes. Thus we have conclusion (2) of the theorem.

Next we turn our attention to conclusion (3) and assume that $m=8$. Using (2) we have all the points of $L \cap H$ in two parallel hyperplanes $K$ and $K^{\prime}$. Without loss of generality we may assume that there are at least five points in $K$. Applying Corollary 1 we know what lattice configurations are possible in $K$, and we now examine these possibilities. Let $Q$ denote the convex hull of the points $O, X_{1}, X_{2}, \cdots, X_{m}$.

Suppose that $K$ has exactly five points of $L \cap H$ in the 5-configuration, then we label the points $O, X_{1}, X_{2}, X_{1}+X_{2}, X_{3}$. These points determine a 3-dimensional face $F$ of $Q$. The 2-dimensional face determined by $O, X_{1}, X_{2}$ is shared by an adjoining 3-dimensional face of $Q$, which must contain a point, say $X_{4}$, of $L \cap H \cap K^{\prime}$. Let $\pi_{1}$ denote the hyperplane containing this 3-dimensional face. Then, applying conclusion (2) again, the remaining points must lie in a hyperplane $\pi_{1}^{\prime}$ parallel to $\pi_{1}$. Similarly, we obtain the hyperplanes $\pi_{2}$ and $\pi_{2}^{\prime}$ starting with the points $O, X_{1}, X_{3}$ and the hyperplanes $\pi_{3}$ and $\pi_{3}^{\prime}$ starting with the points $O, X_{2}, X_{3}$. The set theoretic union of each of these six hyperplanes intersected with $K^{\prime}$ is the boundary of a parallelepiped $P$ that is congruent, with respect to $L$, to the one determined by the points $O, X_{1}, X_{2}, X_{1}+X_{2}, X_{3}$, $X_{1}+X_{3}, X_{2}+X_{3}$, and $X_{1}+X_{2}+X_{3}$. Moreover, the points of $L \cap H \cap K^{\prime}$ must lie at the vertices of $P$.

Let $Y$ be the vertex of $P$ congruent to $O$, with respect to $L$, then the vertices of $P$ are given by $Y, Y+X_{1}, Y+X_{2}, Y+X_{1}+X_{2}, Y+X_{3}, Y+X_{2}+X_{3}$, $Y+X_{1}+X_{3}$, and $Y+X_{1}+X_{2}+X_{3}$. Let $F$ denote the rectangular face of $P$ determined by the first four points and $F^{\prime}$ the rectangular face opposite $F$ determined by the last four points. Now there must be four points of $L \cap H$ in $K^{\prime}$. If more than two of the points are vertices of $F$, then the four vertices of $F$ are in $L$ and we have a 3-dimensional rectangular sublattice generated by $X_{1}, X_{2}, Y$. The same argument holds for the face $F^{\prime}$, therefore we assume at most two points in either face. Thus there must be two of the four points in each face. Moreover they must be adjacent vertices. Since $X_{3}$ is not perpendicular to $X_{1}$ and $X_{2}$, some of the possibilities are clearly impossible. Suppose $Y$ and $Y+X_{2}$ of $F$ are in $L$, then $Y+X_{3}$ must be another point, but to get the fourth point we must use $Y+X_{1}$ or $Y+X_{1}+X_{2}$, but then we will have five points in $K^{\prime}$. If $Y$ and $Y+X_{1}$ of $F$ are in $L$, then again to get another point we must use $Y+X_{3}$, and again have five points in $K^{\prime}$. If $Y+X_{1}$, and $Y+X_{1}+X_{2}$ of $F$ are in $L$, then $O, X_{1}, X_{2}, X_{1}+X_{2}, Y+X_{1}$ and $Y+X_{1}+X_{2}$ are in a hyperplane in the 6-configuration and the remaining points are in a hyperplane parallel to this one through $X_{3}$. When we have the 6-configuration in a hyperplane $R$ and exactly three points in a parallel hyperplane, then all three points cannot be vertices of any one of the three rectangles in the translation of the 6-configuration to the 
second hyperplane. Therefore the three points must be vertices of one of the triangles, and the triangle is either congruent to a triangle in $K$ or it is congruent to the triangle obtained by reflecting the vertices of the triangle in $R$ through the center of the 2-dimensional cross section of $H$ containing the triangle. If $Y+X_{2}$ and $Y+X_{1}+X_{2}$ are in $L$, then $O, X_{1}, X_{2}, X_{1}+X_{2}, Y+X_{2}$, and $Y+X_{1}+X_{2}$ are in a hyperplane in the 6-configuration and again the remaining points are in a parallel hyperplane through $X_{3}$. Thus we have exhausted all the possibilities for this case.

Suppose now that $K$ has six points of $L \cap H$. If they have the 6-configuration, there is nothing to prove. Therefore suppose they have the rhombus configuration. We label the six points $O, X_{1}, X_{2}, X_{1}+X_{2}, X_{3}, X_{1}+X_{2}-X_{3}$. The 2-dimensional face of $Q$ determined by $O, X_{1}, X_{3}$ is shared by an adjoining 3-dimensional face that contains a point of $K^{\prime}$. Let $\pi_{1}$ denote the hyperplane containing this face, then, as before, the remaining points of $L \cap H$ are in a parallel hyperplane $\pi_{1}^{\prime}$. Similarly, starting with the points $O, X_{2}, X_{3}$ we obtain the parallel hyperplanes $\pi_{2}$ and $\pi_{2}^{\prime}$. For $O, X_{1}, X_{1}+X_{2}-X_{3}$ we obtain the hyperplanes $\pi_{3}$ and $\pi_{3}^{\prime}$, and for $O, X_{2}, X_{1}+X_{2}-X_{3}$ the hyperplanes $\pi_{4}$ and $\pi_{4}^{\prime}$. The set theoretic union of these eight hyperplanes intersected with $K^{\prime}$ is the boundary of a rhombus $P$ that is congruent with respect to $L$ to the one in $K$. Moreover, the lattice points in $K^{\prime} \cap H$ must be vertices of $P$.

Let $Y$ be the vertex of $P$ congruent to $O$, then the vertices of $P$ are given by $Y, Y+X_{1}, Y+X_{2}, Y+X_{1}+X_{2}, Y+X_{3}, Y+X_{1}+X_{2}-X_{3}$. There are three 2-dimensional rectangular cross sections of $P$, and the three points of $L \cap K^{\prime}$ cannot all lie in any one of these rectangles, for we would then have four points of $L$ in $K^{\prime} \cap H$. Therefore the three points must be the vertices of a triangular face of $P$. Suppose the points are $Y, Y+X_{2}, Y+X_{3}$, then these three points along with $O, X_{2}$, and $X_{3}$ give us the 6-configuration in a hyperplane, and the remaining points in a parallel hyperplane. If $Y+X_{1}+X_{2}, Y+X_{2}$, $Y+X_{3}$ are the points then these points with $X_{1}+X_{2}, X_{2}, X_{3}$ give us the 6-configuration in a hyperplane and the remaining points are in a parallel hyperplane. If the points are $Y, Y+X_{1}, Y+X_{3}$, then these points with $O, X_{1}, X_{3}$ give the 6-configuration and a parallel hyperplane. If $Y+X_{3}, Y+X_{1}+X_{2}, Y+X_{1}$ are the points, then these points with $X_{1}+X_{2}, X_{1}, X_{3}$ yield the desired result. The triangular faces in the other half of $P$ yield similar results, and we have conclusion (3).

Finally we consider conclusion (4) where $m \geqq 9$, and we have 10 or more points of $L$ on $H$. Again we assume at least 5 points in $K$. First suppose we have exactly five points in $K$, then we obtain the parallelepiped $P$ in $K^{\prime}$ as above. There must be five or more points in $P$, but it is impossible to have more than five, thus there must be exactly five points. Since we have five points in $P$, we must have the vertices of $F$ or $F^{\prime}$ in $L \cap K^{\prime} \cap H$, and we have a rectangular sublattice. If we have six points in $K$ in the 6-configuration, then to have four 
points in $H \cap L \cap K^{\prime}$ we must use the four vertices of one of the two rectangles in $P$, and we have a rectangular sublattice. If we have six points in $K$ in the rhombus configuration, then $P$ must have at least four points, but to put the fourth point in we must use at least three vertices of one of the rectangles therefore we must have all four vertices. The vertices of this rectangle and the one parallel to it in $K$ then give us the eight points and the rectangular sublattice. This concludes the proof of the theorem.

\section{BIBLIOGRAPHY}

1. G. Voronoï, Recherches sur les paralleloedres primitifs, J. Reine Angew, Math. 136 (1909), 67-181.

2. H. Minkowski, Diophantische Approximationen, Teubner, Leipzig, 1907.

3. E. Brunngraber, Über Punktgitter, Dissertation, Wien, 1944.

4. K. H. Wolf, Über kritische Gitter im vierdimensionalen Raum $\left(R_{4}\right)$, Monatsh. Math. 58 (1954), 38-56.

UNIVERSITY OF SOUTH FLORIDA,

TAMPa, FLoRida 